Research Article

\title{
Relationship between Urinary AD7c-NTP with Cerebral Microbleeds Based on APOE Genotype
}

\author{
Yong-peng Yu (iD) and Ya-li Zheng (D) \\ Department of Neurology, Weihai Central Hospital Affiliated to Qingdao University and Weifang Medical College, Weihai, \\ Shandong 264400, China \\ Correspondence should be addressed to Yong-peng Yu; yypeng6688@126.com
}

Received 14 August 2020; Revised 27 June 2021; Accepted 21 September 2021; Published 8 October 2021

Academic Editor: Vida Demarin

Copyright (c) 2021 Yong-peng Yu and Ya-li Zheng. This is an open access article distributed under the Creative Commons Attribution License, which permits unrestricted use, distribution, and reproduction in any medium, provided the original work is properly cited.

\begin{abstract}
Objective. This study was performed to investigate the association between urinary Alzheimer-associated neuronal thread protein (AD7c-NTP) with cerebral microbleeds (CMBs) based on the apolipoprotein E (APOE) genotypes. Methods. A total of 471 patients with acute cerebral infarction screened by magnetic sensitive imaging were enrolled in this study. Among them, twenty-seven cases of mixed CMBs were excluded. A total of 444 patients were divided into two groups according to the presence or absence of CMBs: CMBs group $(n=92)$ and noncerebral microbleeds group (nCMBs) $(n=352)$. Urine AD7cNTP levels were measured using a human enzyme immunoassay kit. Results. In patients with lobar CMBs, there was an interaction between urine AD7c-NTP levels and APOE genotypes $(p=0.01)$. In patients with $A P O E \varepsilon 3 / \varepsilon 3$ allele, the odds ratio of lobar CMBs per standard deviation of urinary AD7c-NTP levels was 0.92 (95\% CI: 0.70-1.19). In patients with $A P O E \varepsilon 2+$ or $\varepsilon 4+$ allele, the multivariate-corrected odds ratio of lobar CMBs per standard deviation of urinary AD7c-NTP levels was 2.95 (95\% CI: 1.38-6.27). Conclusion. A higher level of urinary AD7c-NTP is involved in lobar CMBs, not deep CMBs.
\end{abstract}

\section{Introduction}

Cerebral microbleeds (CMBs) are small cerebral vascular lesions characterized by microbleeds [1]. The occurrence and significance of CMBs in various cerebrovascular diseases are increasingly being paid more attention and have gradually become an important issue in the current research field of cerebrovascular diseases. At present, genetic factors are considered to be involved in the occurrence and development of cerebral vascular disease including CMBs. In terms of genetics, the apolipoprotein $\mathrm{E}(A P O E) \varepsilon 4$ allele is by far the only genetic factor known to increase the risk of $\mathrm{CMBs}$ [2-4]. In 2008, an epidemiological study of CMBs in Iceland found that the APOE $\varepsilon 4 / \varepsilon 4$ genotype was associated with the occurrence of CMBs [5]. De La Monte and Wands further found that $A P O E \varepsilon 4$ was closely related to lobar CMBs [6]. Baseline grading of white matter hyperintensity, lacunar infarction, and $A P O E \& 2$ carrier status can predict CMBs events [7]. Patients with cerebral amyloid angiopathy
(CAA) have a higher incidence of CMBs in the lobe, and $A P O E \& 4$ carriers are more likely to have multiple lobar CMBs at baseline [8]. The study found that the positive rate of $A P O E \varepsilon 2$ in CMBs was higher. Both the APOE $\varepsilon 2$ and $A P O E \& 4$ alleles were associated with increased cortical CMBs [9] and white matter hyperintensity (WMH) load [10]. There is no report on the association between $A P O E$ genotypes and CMBs in the Chinese Han population.

A number of studies have suggested that AD7c-NTP becomes recognized as an effective biomarker for Alzheimer's disease (AD) [6]. There is an association between CMBs and AD [11]. Studies have shown that multiple CMBs or lobar and deep CMBs are associated with an increased risk of all-cause dementia [12]. Multiple lobar CMBs are involved in the rapid progression of dementia and cerebral hemorrhage [13]. The decline in cognitive abilities in patients with both vascular disease and $\mathrm{AD}$ is more severe than that in patients with $\mathrm{AD}$ [14]. It is speculated that $\mathrm{AD} 7 \mathrm{c}-\mathrm{NTP}$, as an effective biomarker of $\mathrm{AD}$, may be closely 
related to CMBs. Whether AD7c-NTP can be used as a biomarker for CMBs has not been studied. It remains unclear whether the AD7c-NTP levels interact with the APOE genotype. Different locations of CMBs may predict different causes and mechanisms. The lobar CMBs are mainly due to CAA, while deep or subcortical CMBs are mainly due to hypertensive microangiopathy such as lipid hyaline degeneration and fibrinoid necrosis of small vessels. Deep cerebral hemorrhage and CMBs may have the same basis of microangiopathy, and cerebral lobe hemorrhage and CMBs are due to CAA. Therefore, based on the APOE genotype, the correlation between urinary AD7c-NTP with CMBs was evaluated to provide effective biomarker for CMBs.

\section{Materials and Methods}

2.1. Study Population. All subjects with acute cerebral ischemic stroke enrolled in this study came from the Huangdao Branch of the Affiliated Hospital of Qingdao University and Weihai Central Hospital Affiliated to Qingdao University from August 2014 to August 2017. Inclusion criteria are as follows: (1) All cases were diagnosed in accordance with the Acute Ischemic Stroke Diagnosis and Treatment Guideline [15]. Cranial magnetic resonance imaging (MRI) confirmed a new infarction (high signal and low apparent diffusion coefficient in DWI sequence). (2) The stroke onset is less than $48 \mathrm{~h}$. Exclusion criteria are as follows: (1) intracranial hemorrhage, brain trauma, hemorrhagic transformation after cerebral infarction, infection, and occupying lesions; (2) patients with severe heart, liver, kidney, pulmonary thrombocytopenia, or gastrointestinal bleeding and severe dementia or Parkinson's disease and Parkinson's syndrome; (3) age > 80 years old; (4) nervous system demyelinating diseases such as Guillain-Barre syndrome and multiple sclerosis; and (5) disturbance of consciousness. All of these were independently assessed and judged by two senior specialists. MRI (including T1W1, T2W1, DWI, and SWI) sequence tests were performed on all patients. Routine ECG, echocardiography, carotid artery ultrasound, brain MRA (or CTA), and TCD examinations were performed. The etiological classification was based on TOAST criteria. The risk factors were recorded including age, gender, smoking, alcohol consumption, Montreal Cognitive Assessment (MOCA) score, National Institutes of Health stroke scale (NIHSS) score, hypertension, systolic blood pressure, diabetes mellitus, hyperlipidemia, and antihypertensive, anticoagulant, and antiplatelet therapy use as well as thrombolysis treatment. This study has been approved by the Ethics Committee of Qingdao University. The study flow chart is showed in Figure 1.

2.2. Urinary Alzheimer Disease Neurofilament Protein Detection. $10 \mathrm{ml}$ of fasting venous blood was collected from all patients (i.e., within 72 hours of onset). Of these, $5 \mathrm{ml}$ of blood was used for blood glucose, blood lipids, and blood routine and routine blood clotting tests. The middle of the morning urine $10 \mathrm{ml}$ from the patients was stored at $2-8^{\circ} \mathrm{C}$ for the detection of AD7c-NTP. A diagnostic kit for AD7cNTP (enzyme-linked immunosorbent assay) was used (the kit was from Shenzhen Anqun Bioengineering Co., Ltd.). The specific operation steps follow the instructions.

2.3. APOE Genotyping. Primers used for APOE genotyping were designed and provided by Nanjing Dongji Biotechnology Co., Ltd. In brief, DNA fragments were amplified separately, using the following primer pairs: $5^{\prime}$-TGTCCAAGG AGCTGCAGG-3' and 5'-CTGCCCATCTCCTCCATCC$3^{\prime}$ for APOE rs429358r and $5^{\prime}$-ATGCCGATGACCTGCA $\mathrm{GAA}^{\prime}$ and $5^{\prime}$-CTGCCCATCTCCTCCATCC- ${ }^{\prime}$ for $A P O E$ rs7412. It is given that $A P O E \varepsilon 4$ and $A P O E \varepsilon 2$ alleles are associated with a high risk of intracerebral hemorrhage and an increase of CMBs and WMH load. Different APOE genotypes may affect different imaging phenotypes of CMBs. Therefore, this study refers to APOE genotyping thoughts in previous studies. According to the different impact of $A P O E$ genotype on $\mathrm{CMBs}$, they are divided into two categories: $\varepsilon 2$ or $\varepsilon 4$ allele $(\varepsilon 2 / \varepsilon 2, \varepsilon 2 / \varepsilon 3, \varepsilon 2 / \varepsilon 4, \varepsilon 3 / \varepsilon 4$, and $\varepsilon 4 / \varepsilon 4)$ carriers and only $\varepsilon 3 / \varepsilon 3$ allele carriers [10].

2.4. Neuroimaging Analysis. GE's superconducting magnetic resonance imaging system (model: GE MR Discovery 750 3.0T) was used to obtain transverse axial T1WI and T2WI, sagittal T1WI, DWI, 3D-TOF MRA, and SWI images. The scanning parameters were FLAIR-T1WI (TR/TE, $1750 \mathrm{~ms} / 24 \mathrm{~ms}$, TI $780 \mathrm{~ms}$; FOV $24 \mathrm{~cm} \times 24 \mathrm{~cm}$, matrix $320 \times 256$ ), FRFSE-T2WI (TR/TE, $4300 \mathrm{~ms} / 95 \mathrm{~ms}$, FOV $24 \mathrm{~cm} \times 24 \mathrm{~cm}$, matrix $512 \times$ 512), and DWI-EPI (TR/TE, $3000 \mathrm{~ms} / 70 \mathrm{~ms}$; FOV $24 \mathrm{~cm} \times$ $24 \mathrm{~cm}$, matrix $160 \times 160$. The SWI (SWAN) sequence parameters are 3D T1-FFE, TR minimum, TE $45.0 \mathrm{~ms}$, inversion angle of $15^{\circ}$, slice thickness of $2 \mathrm{~mm}$, matrix $384 \times 320$, and number of excitations of 1.0. CMBs are defined as uniform low signal areas of oval or circular shape on the SWI sequence, with a diameter of $2-5 \mathrm{~mm}$, no edema surrounding, and not shown on conventional sequences, except for small veins and calcifications. The diagnostic criteria for $\mathrm{CMBs}$ are round or elliptical on the SWI sequence, which has a low signal amplitude of 2 to $10 \mathrm{~mm}$ in diameter, with uniform texture, clear lesion boundaries, and no edema shadow around the lesions, and exclusion of intracranial calcification, iron deposits, cavernous hemangioma, and expanded perivascular space. According to the cerebral microbleeds anatomical rating scale, the CMBs can be divided into deep CMBs (basal ganglia, thalamus, internal capsule, external capsule and corpus callosum, and periventricular white matter), cerebral lobe CMBs (cortical and subcortical white matter), subthecal CMBs (brain stem and cerebellum), and mixed CMBs (cerebral lobe plus deep or subventral plus brain). In order to facilitate the study, the suboccipital CMBs were classified as deep CMBs in the present study. The interference of mixed CMBs was excluded in this study. All MRI images were evaluated by two experienced imaging specialists.

\subsection{Definition of the Main Covariates}

(1) Hypertension: antihypertensive drugs before admission or systolic blood pressure $>140 \mathrm{mmHg}$ or diastolic blood pressure $\geq 90 \mathrm{mmHg}$ at admission 


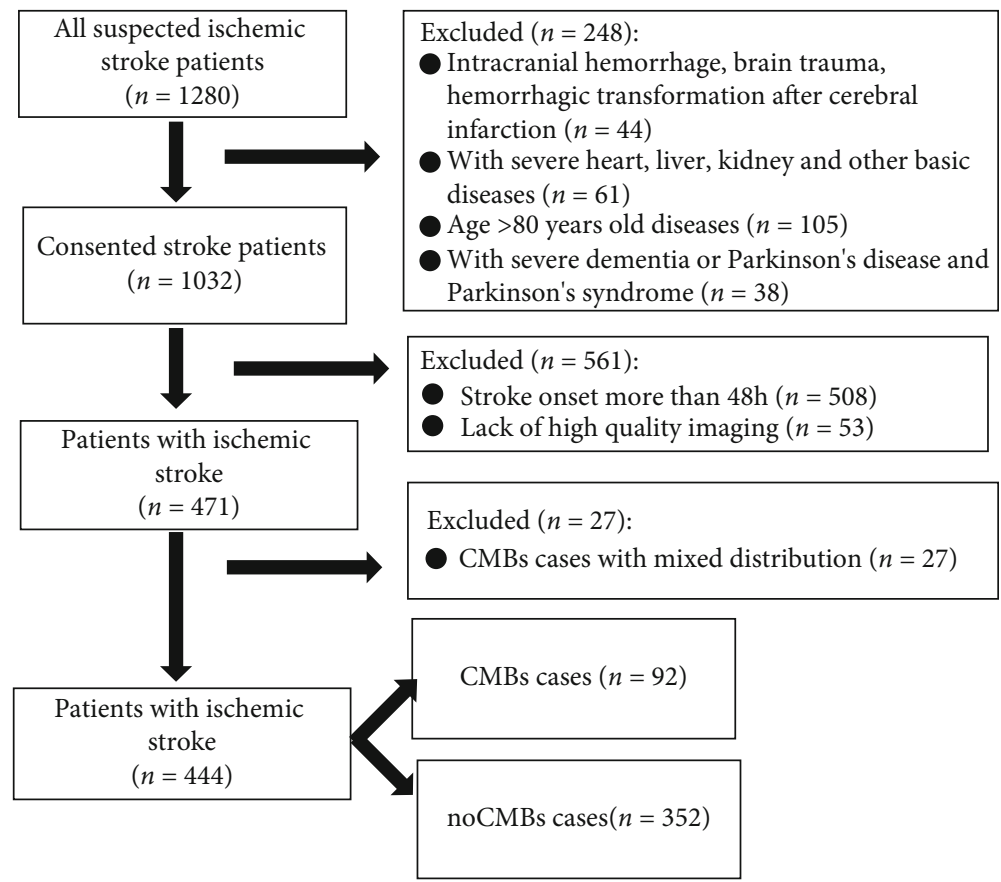

Figure 1: Flow chart of patients enrolled in the study.

(2) Diabetes: history of diabetes; fasting blood glucose $>7.0 \mathrm{mmol} / \mathrm{l}$ or postprandial $2 \mathrm{~h}$ blood glucose $>$ $11.1 \mathrm{mmol} / \mathrm{l}$ combined glycosylated hemoglobin $>$ $6.5 \%$

(3) Hyperlipidemia: past dyslipidemia or hospital admission abnormalities, total cholesterol $>5.72 \mathrm{mmol} / \mathrm{l}$, TG $>1.72 \mathrm{mmol} / \mathrm{l}$, or LDL $>3.12 \mathrm{mmol} / 1$

(4) Smoking: currently smoking or quitting (10 cigarettes/day for more than 5 years)

(5) Drinking: daily alcohol consumption $>50 \mathrm{ml}$, alcohol abstinence or not

2.6. Statistical Analysis. SPSS 16.0 statistical software was used for statistical analysis. Normally distributed measurement data were expressed as the mean \pm standard deviation, and count data were expressed as the percentage (\%). The $t$-test was used to compare the mean of the two samples. The chi-squared test was used to compare two samples of count data. Multivariate comparisons were performed using multiple logistic analysis. $p<0.05$ indicates that the difference was statistically significant. Logistic analysis was used to adjust the confounding factors and analyze the relationship between AD7c-NTP and imaging phenotypes of CMBs and overall CMBs. Different logistic regression models were used for different covariates. The $\varepsilon 2$ and $\varepsilon 4$ alleles are associated with an increased risk of lobar and deep ICH. In this study, the effect of $A P O E$ genotype on the susceptibility of CMBs was different, which was divided into two categories: $\varepsilon 2 / \varepsilon 2, \varepsilon 2 / \varepsilon 3, \varepsilon 2 / \varepsilon 4, \varepsilon 3 / \varepsilon 4$, and $\varepsilon 4 / \varepsilon 4$ and $\varepsilon 3 / \varepsilon 3$. Through analysis of different covariates (age, gender, MOCA score, NIHSS score, hypertension, diabetes, smoking, alcohol, and antiplatelet drugs, anticoagulants, and statin). Interac- tion between APOE genotypes with AD7c-NTP was analyzed. Before the study was conducted, sample estimation and calculation of efficacy were performed. Under normal circumstances, based on previous research data, the prevalence of CMBs in patients with cerebral infarction was estimated between about 20 and 30\%, the lowest value of $20 \%$ to calculate the test efficiency to take 0.8 . It needs about 300-400 sample size.

\section{Result}

3.1. Demographic and Clinical Characteristics of the Study Subjects. This study found that the prevalence of CMBs in the cerebral infarction population was $20.7 \%(92 / 444)$. The majority of patients had single CMBs, of which 42 cases existed multiple CMBs. There were one hundred and nineteen CMBs cases detected including mixed distribution (cerebral lobe plus deep or under the curtain plus lobe) and 27 cases of mixed CMBs, which were excluded in order to avoid the interference to this study. The location of CMBs was more widely distributed, and 21 cases of multiple CMBs were distributed in the brain lobe. The MOCA score in the CMBs group was lower than that in the nCMBs group. Patients with $\mathrm{CMBs}$ from $A P O E \varepsilon 4$ carriers performed worse in terms of cognition (Table 1). Compared with patients with nCMBs, cerebral infarction patients with CMBs had higher $A P O E \varepsilon 2$ or $\varepsilon 4$ allele carrier rates $(p<0.05)$.

3.2. Urinary $A D 7 c-N T P$ Levels and CMBs. At the overall level, there was no difference in urinary AD7c-NTP levels between the nCMBs and CMBs groups $(p>0.05)$. Based on different imaging phenotypic subgroup analyses of CMBs, the level of urinary AD7c-NTP in the lobar CMBs group was significantly higher than that in the $\mathrm{nCMBs}$ group 
TABLE 1: Demographics and clinical characteristics of all participants.

\begin{tabular}{|c|c|c|c|c|}
\hline & No CMBs $(n=352)$ & All CMBs $(n=92)$ & $\begin{array}{c}\text { CMBs } \\
\text { Lobar CMBs }(n=54)\end{array}$ & Deep CMBs $(n=38)$ \\
\hline Age at MRI, mean (SD) & $72(5.4)$ & $74(6.2)$ & $74(5.1)$ & $75(6.7)$ \\
\hline Men, $n(\%)$ & $168(47.8)$ & $48(53.3)$ & $30(55.5)$ & $18(47.3)$ \\
\hline MOCA (median (IQR)) & $27(24-29)$ & $27(25-29)^{\mathrm{a}}$ & $26(23-29)^{\mathrm{b}}$ & $27(25-29)^{\mathrm{c}}$ \\
\hline NIHSS (median (IQR)) & $9(6-15)$ & $9(7-14)$ & $9(6-15)$ & $9(7-16)$ \\
\hline Infarct volume $\left(\mathrm{cm}^{3}\right)$, mean $(\mathrm{SD})$ & $8.5(2.1)$ & $8.0(2.2)$ & $8.2(1.9)$ & $7.8(2.3)$ \\
\hline $\mathrm{SBP}(\mathrm{mmHg})$, mean $(\mathrm{SD})$ & $130(19)$ & $135(20)$ & $133(17)$ & $139(19)$ \\
\hline Diabetes mellitus, $n(\%)$ & $65(18.5)$ & $17(18.5)$ & $10(18.5)$ & $5(13.2)$ \\
\hline HDL cholesterol (mg/dl) & $56(16)$ & $52(18)$ & $48(17)$ & $54(19)$ \\
\hline LDL cholesterol (mg/dl) & $131(33)$ & $140(36)$ & $138(37)$ & $142(35)$ \\
\hline Current smokers, $n(\%)$ & $24(6.8)$ & $7(7.6)$ & $5(9.2)$ & $3(7.9)$ \\
\hline Alcohol, $n(\%)$ & $43(12.2)$ & $13(14.1)$ & $9(16.7)$ & $7(18.4)$ \\
\hline Hypertension, $n(\%)$ & $172(48.9)$ & $58(63.0)^{\boldsymbol{\Lambda}}$ & $31(57.4)$ & $28(73.8)^{\boldsymbol{\Lambda \Lambda}}$ \\
\hline Hypertension treatment, $n(\%)$ & $140(81.4)$ & $45(77.5)$ & $23(74.2)$ & $22(78.6)$ \\
\hline Aspirin use, $n(\%)$ & $200(56.8)$ & $60(65.2)$ & $36(66.6)$ & $22(57.9)$ \\
\hline Anticoagulant use, $n(\%)$ & $7(1.9)$ & $3(3.3)$ & $1(1.8)$ & $2(5.2)$ \\
\hline Thrombolysis treatment & $45(12.7)$ & $12(13.0)$ & $8(14.8)$ & $6(15.7)$ \\
\hline Statin use, $n(\%)$ & $140(39.8)$ & $40(43.5)$ & $23(42.6)$ & $17(44.7)$ \\
\hline \multicolumn{5}{|l|}{ TOAST $(\%)$} \\
\hline LAA & $181(51.4)$ & $38(41.3)$ & $23(42.5)$ & $15(39.5)$ \\
\hline $\mathrm{CE}$ & $8(3.0)$ & $3(4.3)$ & $2(4.1)$ & $1(5.0)$ \\
\hline SAD & $120(45.5)$ & $37(53.6)$ & $26(53.0)$ & $11(55.0)$ \\
\hline \multicolumn{5}{|l|}{ APOE status, $n(\%)$} \\
\hline$\varepsilon 3 / \varepsilon 3, n(\%)$ & $270(76.6)$ & $59(64.1)$ & $34(62.9)$ & $25(65.8)$ \\
\hline$\varepsilon 2 / \varepsilon 2, \varepsilon 2 / \varepsilon 3, \varepsilon 2 / \varepsilon 4, \varepsilon 3 / \varepsilon 4, \varepsilon 4 / \varepsilon 4, n(\%)$ & $82(23.4)$ & $33(35.9)^{*}$ & $20(37.1)^{* *}$ & $13(34.2)^{* * *}$ \\
\hline AD7c-NTP (ng/dl), mean (SD) & $1.05(1.0)$ & $1.21(1.08)^{\mathrm{a}}$ & $1.30(1.11)^{\mathrm{b}}$ & $1.08(1.03)^{\mathrm{c}}$ \\
\hline
\end{tabular}

MOCA: ${ }^{\mathrm{a}} t=2.53, p=0.005 ;{ }^{\mathrm{b}} t=2.34, p=0.009 ;{ }^{\mathrm{c}} t=1.03, p=0.15$. APOE status: ${ }^{*} \chi^{2}=6.0, p=0.01 ;{ }^{* *} \chi^{2}=4.69, p=0.03 ;{ }^{* * *} \chi^{2}=2.21, p=0.14$. AD7c-NTP: ${ }^{\mathrm{a}} t=1.34, p=0.08 ;{ }^{\mathrm{b}} t=1.68, p=0.04 ;{ }^{\mathrm{c}} t=0.17, p=0.43$. Hypertension (total CMBs vs. nCMBs): ${ }^{\mathbf{a}} \chi^{2}=5.87, p=0.016$. Hypertension (lobar CMBs vs. deep CMBs): ${ }^{\wedge} \chi^{2}=2.56, p=0.11$. LAA: large artery atherosclerosis; CE: cardiogenic brain embolism; SAD: small artery disease; APOE: apolipoprotein E; NIHSS: National Institutes of Health stroke scale.

$(p<0.05)$. The urine AD7c-NTP levels in the APOE $\varepsilon 2$ positive or $\varepsilon 4$ positive patients were higher than those in the $A P O E \varepsilon 3 / \varepsilon 3$ genotype carriers $(p<0.05)$ in both $C M B s$ and nCMBs patients. Urinary AD7c-NTP levels were associated with cerebral lobar CMBs (OR: 1.83, 95\% C1: 1.21-3.95). In patients with cerebral lobar CMBs, there was an interaction between urine AD7c-NTP levels and APOE genotype $(p=0.01)$. That is to say, $A P O E$ genotype might be involved in the effect of urine AD7c-NTP on the risk of lobar CMBs. In patients with $A P O E \varepsilon 3 / \varepsilon 3$, the odds ratio for urinary AD7c-NTP levels increased by one standard deviation for cerebral lobe CMBs was 0.92 (95\% CI: 0.70-1.19; $p=0.95$ ). In patients with $A P O E \varepsilon 2$ positive or $\varepsilon 4$ positive carriers, the multivariate-adjusted OR for lobar $\mathrm{CMBs}$ was 2.95 (95\% CI: 1.38-6.27; $p=0.005$ ) (Table 2).

\section{Discussion}

The incidence of CMBs was $20.7 \%$ in the present study, while the incidence fluctuates between $19.4 \%$ and $68.5 \%$ in a previous study [2]. The incidence of CMBs in patients with cerebral hemorrhage is about $38 \%$ to $66 \%$, and cerebral infarction is about $21 \%$ to $26 \%$, and in healthy people, it is about $5 \%$ to $6 \%$. The incidence of CMBs in the Asian population is higher [16]. CMBs are independent risk factors for hemorrhagic transformation after acute cerebral infarction, and they are also important factors for the symptomatic hemorrhagic transformation of patients with cerebral infarction undergoing thrombolysis and anticoagulant therapy [17]. Our study observed that the prevalence of male CMBs was higher than that of females, which may be related to the older age of males in this study. For drug treatment could affect the occurrence of CMBs [16], drug use information was included in statistical analysis.

4.1. AD7c-NTP and the Distribution of CMBs. Urinary AD7c-NTP is associated with overall CMBs in this study. There is a relation between urine AD7c-NTP with the distribution of CMBs. That is, high levels of urinary AD7c-NTP are associated with lobar CMBs, not deep brain CMBs. Urine AD7c-NTP had high specificity and moderate sensitivity in predicting amyloid beta $(A \beta)$ deposition among 
TABLE 2: Interaction between APOE genotype and AD7c-NTP levels in the presence of CMBs.

\begin{tabular}{|c|c|c|c|c|c|}
\hline Model & $A P O E$ & $N$ & $\begin{array}{c}\text { Urine AD7c-NTP } \\
\text { OR }(95 \% \text { CI })\end{array}$ & $p$ & Interaction \\
\hline \multicolumn{6}{|c|}{ Total CMBs } \\
\hline \multirow{2}{*}{1} & 33 & 59 & $0.84(0.54-1.31)$ & 0.44 & \multirow[t]{2}{*}{0.49} \\
\hline & $22,23,24,34,44$ & 33 & $1.05(0.53-1.32)$ & 0.68 & \\
\hline \multirow{2}{*}{2} & 33 & 59 & $0.90(0.60-1.33)$ & 0.60 & \multirow{2}{*}{0.45} \\
\hline & $22,23,24,34,44$ & 33 & $1.03(0.94-1.12)$ & 0.83 & \\
\hline \multicolumn{6}{|c|}{ Lobar CMBs } \\
\hline \multirow{2}{*}{1} & 33 & 34 & $0.83(0.40-1.69)$ & 0.61 & \multirow[t]{2}{*}{0.03} \\
\hline & $22,23,24,34,44$ & 20 & $2.20(1.21-3.97)$ & 0.009 & \\
\hline \multirow{2}{*}{2} & 33 & 34 & $0.92(0.70-1.19)$ & 0.95 & \multirow[t]{2}{*}{0.01} \\
\hline & $22,23,24,34,44$ & 20 & $2.95(1.38-6.27)$ & 0.005 & \\
\hline \multicolumn{6}{|c|}{ Deep CMBs } \\
\hline \multirow{2}{*}{1} & 33 & 25 & $1.04(0.95-1.13)$ & 0.78 & \multirow[t]{2}{*}{0.32} \\
\hline & $22,23,24,34,44$ & 13 & $1.63(0.94-2.7)$ & 0.08 & \\
\hline \multirow{2}{*}{2} & 33 & 25 & $1.05(0.90-1.22)$ & 0.53 & \multirow[t]{2}{*}{0.20} \\
\hline & $22,23,24,34,44$ & 13 & $1.56(0.96-2.08)$ & 0.07 & \\
\hline
\end{tabular}

Odds ratio (OR) of CMBs per standard deviation change in AD7c-NTP (1SD = 1.72 for women, 1.80 for men). Model 1 adjusted for age and sex. Model 2 adjusted for age, sex, aspirin use, anticoagulant use, statin use, diabetes, APOE status ( $\varepsilon 3 / \varepsilon 3$ versus $\varepsilon 2 / \varepsilon 2, \varepsilon 2 / \varepsilon 3, \varepsilon 2 / \varepsilon 4, \varepsilon 3 / \varepsilon 4, \varepsilon 4 / \varepsilon 4)$, and systolic blood pressure.

patients with cognitive impairment [18]. Lobar CMBs in Alzheimer's disease $(\mathrm{AD})$ are associated with cerebral amyloid angiopathy (CAA) due to vascular $\mathrm{A} \beta$ deposits [19]. Deposition of amyloid appears aggravated in patients with cerebral small-vessel disease, especially in $A P O E \varepsilon 4$ carriers [20]. CAA is commonly seen in patients with $A D$, which is associated with parenchymal amyloid and may lead to WMH, microinfarcts, and MBs [21]. Furthermore, we found that urinary $\mathrm{AD} 7 \mathrm{c}-\mathrm{NTP}$ has certain interactions between different CMBs imaging phenotypic subgroups and APOE genotypes. High levels of urinary AD7c-NTP are the risk of lobar CMBs. It suggested that the APOE genotype and urine AD7c-NTP and other biomarkers involved in susceptibility risk control of CMBs were complex. The influence of different $A P O E$ allele states on the association of urinary AD7c-NTP and CMBs with different imaging phenotype risks may be mediated by different mechanisms. This mechanism may play an important role in the pathology of hypertensive cerebral vascular disease represented by deep brain CMBs.

Different imaging phenotypes of CMBs might mean different mechanisms for its occurrence. Our result also showed that the phenotype of CMBs and its susceptibility may have $A P O E$ allele risk dependence. The current mechanism for the development of CMBs still remains unclear, which includes many hypotheses: blood-brain barrier impairment, endothelial cell damage, deposition of beta amyloid, hypoperfusion impairment, inflammatory reactions, and genetic polymorphisms. A previous study found that urinary AD7c-NTP levels were significantly elevated in patients with mild cognitive impairment (MCI) with the $A P O E \varepsilon 4$ genotype [22]. In the context of APOE genotyping in the Han nationality, it is still unclear whether the phenotypic imaging classification of CMBs is related to urinary
AD7c-NTP. It is speculated that urinary AD7c-NTP may serve as a biomarker for CMBs. Based on this hypothesis, this study first investigated the relationship between urinary AD7c-NTP and CMBs based on APOE genotype.

4.2. APOE Genotype Involved in the Risk Effect of Urinary $A D 7 c-N T P$ for CMBs. It is generally believed that the increased risk of disease in $A P O E \varepsilon 4$ carriers is attributed to higher lipid levels. However, increasingly more research evidence shows that the APOE genotype has a direct or indirect effect on the absorption of microglial cells and microglia activation. APOE genotypes have different effects on mitochondrial protein expression, which may be the basis for the susceptibility of different genotypes [23]. It indicated that different $A P O E$ genotypes have different effects on oxidative stress and other biochemical pathological states, which have different effects on disease susceptibility [24, 25]. Therefore, studying the effect of different APOE genotypes on the regulation of chemokines and cytokines may help to further understand the role of ApoE-mediated cytokine regulation in the pathogenesis of CMBs. At present, the correlation between APOE genotype and urinary AD7c-NTP level expression is rare. In 2015, it was reported that in patients with MCI carrying APOE $\varepsilon 4$ genotype, serum brain-derived neurotrophic factor (BDNF) was significantly reduced, while urinary $A D 7 c-N T P$ was significantly increased. Both serum BDNF and urine AD7c-NTP have higher positive predictive values and may be MCI-sensitive biomarkers [22]. The present study found that there was no difference in the levels of urinary AD7c-NTP between nCMBs and CMBs at the overall level. In subgroup analysis of different locations of CMBs, urinary AD7c-NTP levels were correlated with $\mathrm{CMBs}$ in the lobes. Urinary AD7cNTP has certain interactions with $A P O E$ genotypes in 
different location CMBs. Specifically, in patients with $A P O E$ $\varepsilon 2+$ or $\varepsilon 4+$, high-level urinary AD7c-NTP is a risk factor for lobar CMBs.

There are several limitations: First, it is a cross-sectional study. It is unclear whether the course of acute ischemic stroke will affect the levels of urinary AD7c-NTP. Therefore, the relationship between urinary AD7c-NTP and CMBs as well as phenotypic imaging classification needs to be treated with caution. In order to minimize the impact of confounding factors, the cranial MRI was completed within 72 hours after the onset of stroke, and at the same time, blood and urine samples were collected and stored for testing. Secondly, for the differences in ethnicity, the results of this study could not be extended to other ethnic groups. Whether there are other unknown regulatory factors affecting the risk association of $A P O E$ genotype/CMBs remains unclear.

\section{Conclusion}

A higher level of AD7c-NTP is related to lobar CMBs. Urine AD7c-NTP may have an APOE genotype-dependent risk effect on CMBs. Urinary AD7c-NTP has certain interactions with $A P O E$ genotypes in different $\mathrm{CMBs}$ imaging phenotypic subgroups. In the patients with $\varepsilon 2$ or $\varepsilon 4$ allele carrier, the evaluation of urinary AD7c-NTP may contribute to the prediction of CMBs.

\section{Data Availability}

The data used to support the findings of this study are available from the corresponding author upon request.

\section{Ethical Approval}

All procedures performed in studies involving human participants were in accordance with the ethical standards of the institutional and/or national research committee and with the 1964 Helsinki declaration and its later amendments or comparable ethical standards. This study has been approved by the Ethics Committee of Qingdao University.

\section{Conflicts of Interest}

All the authors declare that they have no conflict of interest.

\section{Acknowledgments}

This work was supported by Grants from the National Natural Science Foundation of China (81400957) and Special Fund for Qilu Health and Health Leading Talents Training Project (2020-2025). Thanks are due to Zhang Zheng, Ren Weifeng, Liu Lijun, Yuan Rongrong, Yao Qianqian, and Li Xin for their efforts and help in the specimen collection.

\section{References}

[1] C. Cordonnier, "Brain microbleeds," Practical Neurology, vol. 10, no. 2, pp. 94-100, 2010.
[2] M. Loitfelder, S. Seiler, P. Schwingenschuh, and R. Schmidt, "Cerebral microbleeds: a review," Panminerva Medica, vol. 54, no. 3, pp. 149-160, 2012.

[3] M. R. Benedictus, J. D. C. Goos, M. A. A. Binnewijzend et al., "Specific risk factors for microbleeds and white matter hyperintensities in Alzheimer's disease," Neurobiology of Aging, vol. 34, no. 11, pp. 2488-2494, 2013.

[4] S. Sveinbjornsdottir, S. Sigurdsson, T. Aspelund et al., "Cerebral microbleeds in the population based AGES-Reykjavik study: prevalence and location," Journal of Neurology, Neurosurgery, and Psychiatry, vol. 79, no. 9, pp. 1002-1006, 2008.

[5] M. M. F. Poels, M. W. Vernooij, M. A. Ikram et al., "Prevalence and risk factors of cerebral microbleeds: an update of the Rotterdam scan study," Stroke, vol. 41, 10, Supplement 1, pp. S103-S106, 2010.

[6] S. M. De La Monte and J. R. Wands, "The AD7c-NTP neuronal thread protein biomarker for detecting Alzheimer's disease," Journal of Alzheimer's Disease, vol. 3, no. 3, pp. 345-353, 2001.

[7] J. D. C. Goos, W. J. P. Henneman, J. D. Sluimer et al., "Incidence of cerebral microbleeds: a longitudinal study in a memory clinic population," Neurology, vol. 74, no. 24, pp. 1954-1960, 2010.

[8] P. A. Yates, P. M. Desmond, P. M. Phal et al., "Incidence of cerebral microbleeds in preclinical Alzheimer disease," Neurology, vol. 82, no. 14, pp. 1266-1273, 2014.

[9] E. Loehrer, M. A. Ikram, S. Akoudad et al., "Apolipoprotein E genotype influences spatial distribution of cerebral microbleeds," Neurobiology of Aging, vol. 35, no. 4, pp. 899-905, 2014.

[10] S. Schilling, A. L. DeStefano, P. S. Sachdev et al., "APOE genotype and MRI markers of cerebrovascular disease: systematic review and meta-analysis," Neurology, vol. 81, no. 3, pp. 292300, 2013.

[11] A. Charidimou, S. Martinez-Ramirez, A. Shoamanesh et al., "Cerebral amyloid angiopathy with and without hemorrhage: evidence for different disease phenotypes," Neurology, vol. 84, no. 12, pp. 1206-1212, 2015.

[12] K. Miwa, M. Tanaka, S. Okazaki et al., "Multiple or mixed cerebral microbleeds and dementia in patients with vascular risk factors," Neurology, vol. 83, no. 7, pp. 646-653, 2014.

[13] J. Nagasawa, T. Kiyozaka, and K. Ikeda, "Prevalence and clinicoradiological analyses of patients with Alzheimer disease coexisting multiple microbleeds," Journal of Stroke and Cerebrovascular Diseases, vol. 23, no. 9, pp. 2444-2449, 2014.

[14] A. Viswanathan and S. M. Greenberg, "Cerebral amyloid angiopathy in the elderly," Annals of Neurology, vol. 70, no. 6, pp. 871-880, 2011.

[15] E. C. Jauch, J. L. Saver, H. P. Adams et al., "Guidelines for the early management of patients with acute ischemic stroke: a guideline for healthcare professionals from the American Heart Association/American Stroke Association," Stroke, vol. 44, no. 3, pp. 870-947, 2013.

[16] M. W. Vernooij, A. van der Lugt, M. A. Ikram et al., "Prevalence and risk factors of cerebral microbleeds: the Rotterdam Scan Study," Neurology, vol. 70, no. 14, pp. 12081214, 2008.

[17] C. Cordonnier, R. A.-S. Salman, and J. Wardlaw, "spontaneous brain microbleeds: systematic review, subgroup analyses and standards for study design and reporting," Brain, vol. 130, no. 8, pp. 1988-2003, 2007. 
[18] Y. Zhang, Y. Li, R. Wang, G. Sha, H. Jin, and L. Ma, "Elevated urinary AD7c-NTP levels in older adults with hypertension and cognitive impairment," Journal of Alzheimer's Disease, vol. 74, no. 1, pp. 237-244, 2020.

[19] M. Ikeda, S. Kodaira, H. Kasahara et al., "Cerebral microbleeds, cerebrospinal fluid, and neuroimaging markers in clinical subtypes of Alzheimer's disease," Frontiers in Neurology, vol. 12, p. 543866, 2021.

[20] M. I. Kester, J. D. C. Goos, C. E. Teunissen et al., "Associations between cerebral small-vessel disease and Alzheimer disease pathology as measured by cerebrospinal fluid biomarkers," JAMA Neurology, vol. 71, no. 7, pp. 855-862, 2014.

[21] J. M. Olichney, L. A. Hansen, C. R. Hofstetter, J. H. Lee, R. Katzman, and L. J. Thal, "Association between severe cerebral amyloid angiopathy and cerebrovascular lesions in Alzheimer disease is not a spurious one attributable to apolipoprotein E4," Archives of Neurology, vol. 57, no. 6, pp. 869-874, 2000.

[22] C. Wang, Y. Cui, J. Yang et al., "Combining serum and urine biomarkers in the early diagnosis of mild cognitive impairment that evolves into Alzheimer's disease in patients with the apolipoprotein E $\epsilon 4$ genotype," Biomarkers, vol. 20, no. 1, pp. 84-88, 2015.

[23] L. Jiang, J. Zhong, X. Dou, C. Cheng, Z. Huang, and X. Sun, "Effects of ApoE on intracellular calcium levels and apoptosis of neurons after mechanical injury," Neuroscience, vol. 301, pp. 375-383, 2015.

[24] C. COLTON, C. BROWN, D. COOK et al., “_APO_E and the regulation of microglial nitric oxide production: a link between genetic risk and oxidative stress," Neurobiology of Aging, vol. 23, no. 5, pp. 777-785, 2002.

[25] L. L. Zhao, G. Su, L. X. Chen et al., “Apolipoprotein E polymorphisms are associated with ischemic stroke susceptibility in a Northwest China Han population," Bioscience Reports, vol. 37, no. 6, p. 37(6), 2017. 